\title{
DE-RISKING: UNA CONSECUENCIA INDESEADA DEL ENFOQUE BASADO EN EL RIESGO APLICADO POR LOS BANCOS EN MATERIA DE PREVENCIÓN DEL LAVADO DE ACTIVOS Y FINANCIAMIENTO DEL TERRORISMO
}

\author{
DE-RISKING: AN UNINTENDED CONSEQUENCE OF THE RISK- \\ BASED APPROACH APPLIED BY BANKS REGARDING MONEY \\ LAUNDERING AND TERRORIST FINANCING PREVENTION
}

\author{
Ángela Toso Milos*
}

\begin{abstract}
RESUMEN: Durante los últimos años se ha observado que diversos bancos alrededor del mundo han decidido no iniciar, o bien, terminar relaciones comerciales preexistentes, con ciertas categorías de clientes consideradas de mayor riesgo de lavado de activos y/o financiamiento del terrorismo (LA/FT). Esta práctica, denominada de-risking, constituye el objeto de estudio de este trabajo. En el artículo se revisa el contexto en que se ha originado esta práctica, se examinan las principales causas y repercusiones que se le han atribuido en relación con la prevención del LA/FT, se identifican aquellas categorías de clientes afectados por la misma, tanto en Chile como en el extranjero, y se señalan algunas posibles vías de solución a este fenómeno.
\end{abstract}

Palabras clave: lavado de activos, enfoque basado en el riesgo, inclusión financiera, debida diligencia.

ABSTRACT: In recent years, it has been observed that several banks around the world have decided not to initiate, or terminate, pre-existing commercial relationships with clients considered to be of higher risk regarding money laundering and/or terrorist financing (ML/TF). The study of this phenomenon, known as de-risking, is the main objective of this work. The article reviews the context in which this practice has been originated, examines the main causes and repercussions attributed to it in relation to the prevention of ML/TF, as well as those categories of clients affected by it, both in Chile and abroad, and identifies some possible ways of solving this phenomenon.

Key words: money laundering, risk-based approach, de-risking, financial inclusion, due diligence.

\section{INTRODUCCIÓN}

En el marco del diseño e implementación de los modelos de cumplimiento normativo en materia de prevención del lavado de activos y financiamiento del terrorismo (LA/ FT) ${ }^{1}$, se ha evidenciado, durante los últimos años, que diversos bancos -tanto en Chile

\footnotetext{
Doctora en Derecho, Universidad de Salamanca, España. Profesora de Derecho Comercial. Pontificia Universidad Católica de Valparaíso. Dirección postal:Av. Brasil 2950, Valparaíso-Chile. Dirección electrónica: angela. toso@pucv.cl.

1 A partir de lo señalado en Nieto (2015) p. 26 y Alarcón (2016) p. 63, los modelos de cumplimiento normativo o compliance pueden definirse como aquellos sistemas de gestión cuyo objeto es prevenir y, si es necesa-
} 
Toso Milos, Ángela "De-risking: una consecuencia indeseada del enfoque basado en el riesgo ..."

como en el extranjero- han decidido no iniciar, o bien terminar relaciones comerciales preexistentes con ciertas categorías de clientes consideradas de mayor riesgo de LA/FT ${ }^{2}$, dando lugar a un fenómeno conocido como de-risking ${ }^{3}$.

El protagonismo que los bancos han alcanzado en este campo se explica, probablemente, en función de las características específicas que reviste su actividad, que conlleva la realización de un gran número de operaciones, así como el establecimiento de un conjunto variado de relaciones comerciales, lo que implica una considerable exposición a daños legales y reputacionales, entre otros costos derivados del LA/FT. Otro factor que contribuye a explicar dicha práctica es la antigüedad que tienen estas entidades de crédito como sujetos obligados a adoptar modelos de prevención de LA/FT, por lo que cuentan desde hace ya tiempo con sistemas diseñados con un enfoque basado en el riesgo, que les permite disponer de mayor información para tomar decisiones en esta materia.

Se ha señalado que el de-risking surge a partir de una aplicación equivocada de las medidas diseñadas para evitar el LA/FT y que constituye una amenaza para el régimen de prevención de estos delitos. Al respecto, el Grupo de Acción Financiera Internacional $(\mathrm{GAFI})^{4}$ ha indicado que esta práctica podría generar una mayor vulnerabilidad del sistema para ser utilizado con fines ilícitos, toda vez que implica dejar a ciertos clientes al margen de las medidas que se adoptan en este ámbito 5 . Este planteamiento invita a reflexionar acerca de las verdaderas causas del de-risking y sus consecuencias en lo que atañe a la efectividad del régimen de prevención del LA/FT.

En este contexto, el presente artículo tiene por objeto examinar el escenario en que se ha originado el de-risking, las principales causas y repercusiones que se le han atribuido en relación con la prevención del LA/FT, identificar aquellas categorías de clientes afectados por el mismo, tanto en Chile como en el extranjero, y señalar algunas posibles vías de solución a este fenómeno. La hipótesis del trabajo consiste en sostener que el de-risking es un efecto indeseado del enfoque basado en el riesgo que deben aplicar los bancos para la prevención del LA/FT, que ha afectado a diversas categorías de clientes y que puede explicarse, en buena medida, en razón de los costos asociados al cumplimiento de la normativa en esta materia. A su vez, las consecuencias atribuidas a dicha práctica, en el marco de la

rio, identificar y sancionar las infracciones de leyes, regulaciones, códigos o procedimientos internos aplicables, promoviendo una cultura de respeto a las normas al interior de una organización. En particular, los modelos de prevención del LA/FT deben ser adoptados por diversos actores del sistema económico y financiero en nuestro país, señalados taxativamente en el artículo 3 de la Ley 19.913, denominados comúnmente "sujetos obligados". En Chile, respecto de los bancos, y sobre la base de lo establecido por el GAFI, los principales instrumentos normativos que sustentan estos modelos de cumplimiento son: la Ley 19.913, de 2003; diversas circulares de la Unidad de Análisis Financiero; y, el Capítulo 1-14 de la Recopilación Actualizada de Normas (RAN) de la Comisión para el Mercado Financiero, en tanto sucesora legal de la Superintendencia de Bancos e Instituciones Financieras, a partir de lo dispuesto en el artículo 2 del Decreto con Fuerza de Ley No 3, de 1997, modificado recientemente a través de la Ley 21.130, de 2019. Finalmente, los bancos también pueden adoptar el modelo de cumplimiento establecido en el artículo 4 de la Ley 20.393, de 2009.

2 ASBA (2017) p. 4.

3 GAFI (2014).

4 El GAFI elabora las denominadas “40 Recomendaciones”, que constituyen los principales estándares internacionales vigentes en materia de prevención del LA/FT. Al respecto, véase GAFI (2012).

5 GAFI (2014). 
efectividad del régimen de prevención, son difíciles de comprobar, aunque deben ser analizadas para hallar soluciones que permitan equilibrar los diversos intereses que están en juego. Las soluciones que puedan plantearse en este ámbito deberían, por una parte, brindar suficiente seguridad jurídica a los bancos en el cumplimiento de sus deberes y, por otra, proteger a los clientes frente a situaciones de de-risking injustificado.

El estudio del de-risking, a partir de la experiencia desarrollada por los bancos, puede resultar útil para examinar, en general, las consecuencias derivadas de la adopción de cualquier otro modelo de cumplimiento estructurado con un enfoque basado en el riesgo. Por último, cabe hacer presente que, si bien la práctica objeto de este trabajo tiene incidencia en diversos campos $(v \cdot g r$. libre competencia, inclusión financiera, recaudación tributaria, entre otros) el análisis que se realiza aquí está circunscrito al régimen de prevención del LA/FT.

Este artículo cuenta con cuatro apartados. En el primero se examina el contexto en que se ha originado el de-risking, esto es, el enfoque basado en el riesgo con relación al deber que tienen los bancos de aplicar medidas de debida diligencia respecto de sus clientes. En el segundo se vinculan dichas exigencias con la decisión que han tomado estas entidades de crédito en el sentido de practicar el de-risking, analizando sus principales motivos y las consecuencias que se le han atribuido, en el marco del régimen de prevención del LA/FT. En los últimos dos apartados se busca identificar las principales categorías de clientes afectadas por el de-risking, tanto a nivel internacional como en nuestro país, y se revisan algunas propuestas de solución para abordar la problemática que genera esta práctica. Finalmente, se formulan algunas conclusiones surgidas a partir del estudio realizado.

\section{CONTEXTO EN QUE SE DESARROLLA EL DE-RISKING: EL ENFOQUE BASADO EN EL RIESGO DE LA/FT Y LA APLICACIÓN DE LAS MEDIDAS DE DILIGENCIA DEBIDA}

Según se ha señalado, en determinadas ocasiones, los bancos han decidido evitar o terminar relaciones de negocio con ciertas categorías de clientes en función del riesgo de LA/FT que dichos vínculos representan. Este fenómeno conocido, en inglés, como de-risking, ha sido definido indicando que se trata de aquel “...proceso en el cual las instituciones financieras abandonan o reducen significativamente ciertas líneas de negocio para evitar riesgos regulatorios y de cumplimiento"6, e implica una opción deliberada de bancos, en el sentido de evadir los riesgos de LA/FT, en lugar de gestionarlos ${ }^{7}$.

La necesidad de gestionar los riesgos en materia de LA/FT surge a partir de lo establecido por el Grupo de Acción Financiera Internacional (GAFI) y lo señalado en nuestro ordenamiento jurídico, en el sentido que los programas de prevención que adoptan los bancos en este ámbito se han de estructurar sobre la base del riesgo que estos sujetos tienen de verse expuestos a la comisión de dichos delitos ${ }^{8}$. Así, se pretende determinar, a través del conocimiento de los atributos específicos de sus actividades, si estas contribuyen a incre-

\footnotetext{
6 ASBA (2017) p. 4.

7 GAFI (2015a).

8 GAFI (2012) p. 9, Recomendación No 1 y GAFI (2014a) p. 6.
} 
mentar o disminuir el riesgo de LA/FT ${ }^{9}$. Desde esta perspectiva, el GAFI ha entendido que el enfoque basado en el riesgo (EBR) (en inglés, risk-based approach) es el más adecuado para enfrentar la prevención del LA/FT, ya que permitiría equilibrar los diversos intereses que están en juego, alcanzando una mayor eficiencia a través del empleo de los recursos de los bancos en aquellos supuestos especialmente vulnerables al LA/FT ${ }^{10}$.

Para aplicar el EBR se ha entendido que es necesario que los sujetos obligados identifiquen, gestionen y monitoreen los riesgos de LA/FT que afrontan en el ejercicio de su giro. En el marco de la identificación de los riesgos de LA/FT, se debe determinar la probabilidad e impacto que estos representan en las actividades desarrolladas por los sujetos obligados, para lo cual deben elaborar un mapa o matriz de riesgos, que ha de actualizarse periódicamente $^{11}$. Luego, se espera que dichos sujetos obligados adopten las medidas de prevención y reacción que correspondan para mitigar los riesgos identificados ${ }^{12}$. Finalmente, es necesario realizar un seguimiento de las políticas, normas, procedimientos y medidas adoptadas, con la finalidad de formular los ajustes o correcciones necesarias para mejorar el modelo $^{13}$. Es así como la implementación del EBR requiere de importantes esfuerzos -en términos humanos y económicos- por parte de los sujetos obligados, que se incrementan en el caso de aquellos supuestos de mayor riesgo de LA/FT.

Una de las manifestaciones más importantes de lo que se viene señalando, y que está presente en los modelos de prevención del LA/FT, se encuentra en la aplicación de las denominadas "medidas de debida diligencia" (DDC) $)^{14}$, que tienen por objeto la recopilación y análisis continuo de antecedentes de los clientes, relativos a su identificación; el propósito y carácter de la relación comercial que pretenden establecer; sus actividades; y, la individualización del beneficiario final, en su caso ${ }^{15}$. Dicha información debe permitir determinar si el cliente que se trata pretende ejecutar, o ha realizado, alguna operación sospechosa de LA/FT y que, por tal circunstancia, deba reportarse a la Unidad de Análisis Financiero $(\mathrm{UAF})^{16}$. A este respecto, el GAFI indica que ha de exigirse a las instituciones financieras

\footnotetext{
9 Ross y Hannan (2007) p. 110.

10 GAFI (2014a) p. 6.

11 Enseñat (2016) pp. 67-70 y 72; Gómez (2017) pp. 22 y 211; Del Cid (2007) pp. 161-162; GAFI (2014a) p. 9; Carrau (2016) pp. 25 y 43; Neira (2016) p. 471; Artaza (2013) p. 558; Coca (2013) p. 57; GAFI (2007) p. 22; GAFI (2014a) p. 9; Ayala de la Torre (2017) p. 25; Coca (2013) p. 57; Olaya y Sánchez (2017) pp. 22-23; Basel Comitee on Banking Supervision (2016) p. 4; y, López (2017) p. 241.

12 ENSEÑAt (2016) pp. 82-84.

13 Carbayo (2016); López (2017) p. 240; Mariño y otros (2014) p. 11; Artaza (2013) p. 557; Piña (2012) p. 45; Gómez (2017) p. 229; ENSEÑAT (2016) p. 86; y, BRuñen (2017) p. 190.

14 Estas medidas se formulan en las Recomendaciones del GAFI (No 10 a 19), así como en el ordenamiento jurídico chileno, particularmente, en las Circulares de la Unidad de Análisis Financiero (UAF) No 49 y 57, a las que cabe agregar, en el caso de los bancos, lo dispuesto en el Capítulo 1-14 de la Recopilación Actualizada de Normas (RAN) de la CMF.

15 GAFI (2012) p. 14. En Chile, el deber de identificar al beneficiario final se formula en la Circular UAF No 57 y tiene por finalidad evitar la instrumentalización de personas o estructuras jurídicas para el LA/FT.

16 Cabe hacer presente que la UAF no está habilitada para acceder, sin autorización previa, a aquella información de los clientes sujeta a reserva o secreto bancario. Sin embargo, podrá conocer dicha información cuando esta sea incluida en los reportes de operaciones sospechosas que los bancos o la CMF le remitan, en virtud de lo dispuesto en el artículo 3 de la Ley 19.913. Además, en razón de lo indicado en el numeral 35 del artículo 5
} 
que adopten cada una de las medidas de DDC, determinando el alcance de las mismas mediante un enfoque basado en el riesgo ${ }^{17}$, de manera que los Estados deberían formular políticas de DDC que contemplen medidas diferenciadas ("simplificadas", "normales", y "reforzadas") dependiendo del riesgo de LA/FT que representa el cliente u operación que se trata. De modo que, si bien las medidas de DDC deben aplicarse a todos los clientes, hay casos en que es necesario contar con procedimientos más rigurosos de recopilación y verificación de sus antecedentes. Al contrario, habrá otros supuestos en que el riesgo es menor y no será imperioso aplicar procedimientos tan estrictos ${ }^{18}$.

Las medidas de DDC aparecen clasificadas de esta manera en nuestra normativa interna de prevención del LA/FT ${ }^{19}$. Además, dicha normativa señala diversos supuestos en que los bancos han de aplicar medidas adicionales de $\mathrm{DDC}^{20}$. El primer caso de este tipo es el de las "Personas Expuestas Políticamente" (PEPS) ${ }^{21}$, categoría que se ha formulado con la finalidad de evitar el lavado de activos derivado, en general, de actos de corrupción ${ }^{22}$. En estos supuestos, las entidades bancarias deben implementar medidas para determinar si el cliente (o el beneficiario final) es una PEP. Luego, para vincularse con estos clientes se debe obtener la aprobación de la alta gerencia del banco y tomar medidas para determinar la fuente de sus fondos. Por último, es necesario llevar a cabo un monitoreo intensificado de dichas relaciones comerciales ${ }^{23}$. Otra categoría considerada vulnerable al LA/FT por el GAFI, así como por la Comisión para el Mercado Financiero (CMF), son las relaciones de corresponsalía bancaria. Estos vínculos se asientan sobre un principio de confianza entre las entidades de crédito, lo que puede provocar que el banco corresponsal no verifique la identidad ni las características de los clientes del banco representado, confiando en que este último habrá llevado a cabo los controles pertinentes ${ }^{24}$. Es por ello que estas relacio-

del Decreto Ley No 3.538, de 1980, cuyo texto actual fue fijado por medio de la Ley 21.000, de 2018, la CMF debe evaluar la efectividad de los controles implementados por los bancos en materia de prevención del LA/ FT. En aquellos supuestos en que la CMF advierta alguna conducta u omisión que pudiere ser indiciaria de la utilización del sistema financiero y de otros sectores de la actividad económica para el LA/FT, deberá reportar a la UAF, “...comunicándole, además, todos los antecedentes que pudieren serle útiles para iniciar y llevar adelante una investigación respecto de dichas situaciones, incluyendo aquella información a la que se refiere el inciso segundo del artículo 154 de la Ley General de Bancos". Según se señala en la Historia de la Ley 21.130, así se pretende armonizar los legítimos derechos ciudadanos, particularmente, el derecho a la protección de sus datos personales, que podría verse afectado si la UAF accede libremente a los mismos, con la entrega de herramientas a la autoridad para combatir el LA/FT. Biblioteca del Congreso Nacional (2018).

17 GAFI (2012) p. 14.

18 GAFI (2007) p. 9 y Toso (2014) p. 409.

19 Véase Circular UAF No 59.

20 Toso (2014) p. 409.

${ }^{21}$ Denominados, en inglés, Politically Exposed Persons. En Chile, y a partir de lo señalado en GAFI (2012) p. 18, las PEPS están definidas en la Circular UAF No 49 y en el Capítulo 1-14 RAN de la CMF como "...los chilenos o extranjeros que desempeñan o hayan desempeñado funciones públicas destacadas en un país, hasta a lo menos un año de finalizadas las mismas". Las medidas que deben adoptar a este respecto se formulan en el apartado IV de la Circular UAF No 49 y en el apartado II.2 del Capítulo 1-14 de la RAN CMF.

22 Greenberg y otros (2009) p. 13.

${ }^{23}$ GAFI (2012) p. 16.

24 Sánchez (2002) p. 314 y SÁnchez (2006) p. 37. Según se indica en Comité de Supervisión Bancaria de BasiLEA (2001) p. 19 y SEPBLAC (2007) p. 1, la banca de corresponsales (correspondent banking) consiste en la provisión 
nes también deben ser aprobadas por la alta gerencia del corresponsal, sobre la base de la información disponible acerca de la naturaleza de los negocios de la entidad representada, de su reputación y de la calidad de la supervisión que llevan a cabo los reguladores del país en el que está situada. También es necesario evaluar los controles de LA/FT que realiza la institución representada y convencerse acerca de que ha aplicado las medidas de DDC respecto de aquellos clientes que tienen acceso a las cuentas del banco corresponsal, entre otras medidas ${ }^{25}$. Finalmente, pueden concebirse también como operaciones o clientes de mayor riesgo, aquellos ubicados en Estados que no aplican las recomendaciones del GAFI o que lo hacen de manera insuficiente; las transferencias electrónicas de fondos, cuyo riesgo de LA/FT se relaciona con la identificación de sus intervinientes (sobre todo cuando provienen de Estados que poseen una legislación laxa en materia de DDC); y, las demás categorías que el banco determine a partir de la aplicación del $\mathrm{EBR}^{26}$.

De acuerdo con lo que se ha indicado, el régimen jurídico de prevención contempla la aplicación de medidas reforzadas de DDC por parte de los bancos para gestionar y monitorear aquellos vínculos comerciales de mayor riesgo de LA/FT, labor que conlleva un esfuerzo adicional a su cargo. Precisamente, en el contexto de esta tarea dichas entidades de crédito han tomado la decisión, en determinados casos, de evitar los riesgos de LA/FT en vez de gestionarlos, lo que ha dado lugar al de-risking. A continuación, se analizarán los principales motivos y consecuencias asociadas a esta práctica, dando cuenta del hecho que estos no han sido identificados siempre de manera adecuada.

\section{LA DECISIÓN DE NO ESTABLECER O MANTENER RELACIONES DE NEGOCIOS CON DETERMINADAS CATEGORÍAS DE CLIENTES EN FUNCIÓN DEL RIESGO DE LA/FT: SUS MOTIVOS Y CONSECUENCIAS EN EL MARCO DEL RÉGIMEN DE PREVENCIÓN}

A partir de lo señalado por el GAFI y nuestra regulación de prevención, es evidente que el EBR obliga a los bancos a reflexionar en torno a los riesgos de LA/FT que afrontan. En este contexto, es factible que se generen distintas posibilidades que dependen, en definitiva, del denominado "apetito de riesgo" que tenga cada institución financiera, el cual consiste en “... la cantidad de riesgo a nivel global, que los miembros de la alta dirección de una organización están dispuestos a aceptar en la búsqueda de valor” ${ }^{27}$. El nivel de tolerancia es diferente respecto de cada riesgo identificado y se establece en función de su probabilidad e impacto, de las actividades de la organización, de sus valores corporativos y de los costos asociados al cumplimiento de la regulación aplicable ${ }^{28}$.

\footnotetext{
de servicios bancarios por un banco ("corresponsal”) a otro ("representado"). La necesidad de la banca de corresponsales nace cuando una entidad necesita prestar un servicio en un tercer país en el que no tiene presencia física.

25 Apartado II.3., Capítulo 1-14 de la RAN CMF.

26 GAFI (2012) pp. 16-19 y 66-67 y Toso (2014) p. 409.

27 ENSEÑAT (2016) pp. 87 y 127.

28 Enseñat (2016) p. 88; Durner y Shetret (2015) p. 8; y Bruñen (2017) p. 190.
} 
En razón del eventual impacto jurídico, reputacional y, en definitiva, patrimonial que puede provocar para un banco ser sancionado en razón de una implementación inadecuada de su modelo de LA/FT, podría pensarse que la tolerancia a los riesgos de las entidades de crédito en este ámbito es baja y que ello debiera reflejarse en el despliegue de sus mayores esfuerzos para adoptar medidas que permitan mitigarlos. Sin embargo, los bancos, siguiendo el EBR, muchas veces han preferido evitar o terminar relaciones de negocio con ciertas categorías de clientes, esto es, han desarrollado el de-risking ${ }^{29}$. Esta práctica ha afectado, en general, a aquellos clientes u operaciones consideradas más vulnerables al LA/FT, respecto de las cuales tanto el GAFI como nuestra normativa vigente en materia de prevención del LA/FT señalan que los bancos deben implementar medidas adicionales de DDC, a fin de gestionar y monitorear los riesgos involucrados. Atendida esta realidad, el de-risking produce un efecto indeseado y a la vez paradójico en el marco del régimen de prevención del LA/FT. Indeseado porque, desde el momento en que los bancos optan por evitar a estos clientes en lugar de gestionar el riesgo que conllevan, se están apartando de lo que pretende el sistema, que es, justamente, que dichas categorías de clientes estén sometidas a un mayor control por parte de las entidades de crédito. Y paradójico desde la perspectiva del diseño del sistema de prevención del LA/FT, ya que los bancos se deshacen de los riesgos que ciertos clientes representan a partir de los insumos que les proporciona el propio enfoque basado en el riesgo.

Se ha argumentado que el de-risking surge a partir de una aplicación equivocada de las medidas de prevención del LA/FT, en términos tales que dicha práctica ha sido calificada como una "falla del mercado" 30 . Al respecto, el GAFI señala que es necesario entregar a los bancos una mayor orientación en torno al EBR, en el sentido de que se les debe aclarar que esta perspectiva en ningún caso implica la desvinculación de ciertas categorías de clientes, sin considerar el nivel de riesgo asociado a cada uno de ellos en particular. De este modo, los bancos están llamados a terminar con aquellas relaciones comerciales que así lo ameriten en el marco de la prevención del LA/FT, pero sobre la base de un análisis efectuado de manera individual respecto de cada cliente u operación, y solo cuando los riesgos de LA/FT no puedan ser mitigados ${ }^{31}$

Sin embargo, de acuerdo con lo señalado por diversa doctrina especializada a partir de la experiencia verificada en este campo, el principal motivo del de-risking no parece radicar en una comprensión inadecuada del EBR por parte de los bancos, sino más bien en el aumento de los costos asociados al cumplimiento normativo en materia de LA/FT ${ }^{32}$. Ahora bien, se debe considerar que dichos costos se incrementan cuando existe falta de claridad en la regulación aplicable. Así, por ejemplo, en el marco de la adopción de las medidas DDC, se ha referido la existencia de una confusión respecto del alcance de estas, en el sentido de que su adecuado cumplimiento implicaría también, para los sujetos obligados, el deber de identificar a quienes tienen la calidad de clientes de sus clientes (en inglés, know

29 GAFI (2014); Howard (2017a) p. 213; y De KoKer y otros (2017) p. 119.

30 Durner y Shetret (2015) p. 3.

31 GAFI (2014). En este mismo sentido, véanse LeWIs (2016) y Durner y Shetret (2015) p. 3.

32 Véanse, por todos, Howard (2017a) p. 213 y Fuji-Rajani (2017) p. 216. 
your customer's customers), de modo que, al encontrarse los bancos en situaciones en que dicha tarea se torna muy compleja han decidido terminar estas relaciones comerciales ${ }^{33}$. Por otra parte, se ha sostenido que el de-risking se explicaría en virtud del incremento de la presión ejercida por los reguladores, reflejada en las sanciones aplicadas durante los últimos años a los sujetos obligados ante el incumplimiento de sus deberes. Junto con ello, se habría evidenciado un cambio en la percepción de los riesgos por parte de los bancos, que se manifestaría en una mayor conciencia del impacto legal y reputacional que puede significar para estas entidades verse involucradas en casos de LA/FT ${ }^{34}$.

Por lo tanto, las decisiones que han tomado los bancos de abandonar los vínculos que mantienen con determinadas categorías de clientes parecen decir relación, fundamentalmente, con los costos económicos que implica la regulación de prevención del LA/ $\mathrm{FT}^{35}$, tanto desde la perspectiva de la implementación de las medidas y controles que se requieren, como debido a las eventuales sanciones legales y reputacionales a las que pueden verse expuestas las entidades de crédito en estos casos. Luego, cuando se decide practicar el de-risking, se estarían haciendo primar dichos costos frente a los menores beneficios que parece representar para los bancos el cumplimiento de las medidas de prevención ${ }^{36}$.

Aparentemente, los costos vinculados a la aplicación de las medidas DDC se conciben como uno más de aquellos que han de asumir los bancos en la explotación de su giro. Ello permitiría explicar la práctica del de-risking en mercados competitivos, en que las ganancias marginales asociadas a ciertos tipos de operaciones o clientes son bajas, como ha ocurrido, por ejemplo, con las relaciones de corresponsalía bancaria, según se evidenciará en el siguiente apartado de este trabajo. Por otra parte, se ha acusado a los bancos de invocar la dificultad que reviste el cumplimiento de las medidas DDC como una excusa para proteger su industria frente a otros competidores. En Chile, este argumento ha sido invocado por los operadores de criptomonedas ante el Tribunal de Defensa de la Libre Competencia (TDLC), caso que será tratado en el último apartado de este artículo. Al respecto, cabe hacer presente que los operadores de criptomonedas no están obligados a contar con un modelo de prevención del LA/FT y, en consecuencia, no deben asumir los mismos costos que los bancos en este ámbito.

33 GAFI (2016) p. 4 y Thomson Reuters (2018) p. 9.

34 Durner y Shetret (2015) p. 8.

35 Acerca del concepto de costo de regulación (regulatory cost), la OECD (2014) pp. 11-12, ha señalado que este comprende "....all of the costs attributable to the adoption of a regulatory requirement, whether direct or indirect in nature and whether borne by business, consumers, government and its respective authorities (i.e. taxpayers) or other groups". En este sentido, identifica los costos de cumplimiento como una de las categorías de los costos de regulación, definiéndolos como: “... costs that are incurred by businesses or other parties at whom regulation may be targeted in undertaking actions necessary to comply with the regulatory requirements, as well as the costs to government of regulatory administration and enforcement. Compliance costs can be further divided into administrative burdens, substantive compliance costs and administration and enforcement costs". También respecto del concepto de costo de regulación y sus clasificaciones, puede consultarse Marneliffe y Vereek (2011) pp. 341-356. En particular, respecto de los diversos costos que, en la práctica, ha generado la normativa de prevención del LA/FT para los bancos, véase SAPERSTEIN y otros (2015) pp. 4-5 y ThOMSOn Reuters (2018).

36 Geiger y Wuensch (2006) pp. 13-14 y Saperstein y otros (2015) p. 5. 
En cuanto a las consecuencias atribuidas al de-risking en el marco del régimen de prevención del LA/FT, el GAFI ha señalado que, cuanta más cobertura posea un sistema financiero, mayores son las posibilidades de prevenir eficazmente estos delitos. Luego, sostiene que si una persona (natural o jurídica) no tiene acceso al sistema, existen más probabilidades de que utilice canales financieros informales, con lo que se incrementaría el riesgo de LA/FT ${ }^{37}$.

Lo indicado por el GAFI pone en evidencia la necesidad de reflexionar acerca de los reales efectos que el de-risking puede provocar en materia de prevención del LA/FT. En este sentido, es necesario tener presente que la circunstancia de que ciertos clientes sean arrastrados hacia la informalidad por medio del de-risking no significa que estos, necesariamente, vayan a realizar conductas de LA/FT. Por otra parte, la erradicación de esta práctica tampoco permite garantizar que no se cometan dichos delitos, ya que quienes incurren en ellos podrían estar interesados en huir hacia la informalidad si observan que los bancos incrementan sus medidas y controles, por temor a ser descubiertos. En cualquier caso, se trata de cuestiones difíciles de comprobar, respecto de las cuales aún no existe evidencia científica de respaldo.

No obstante, el GAFI parece tener razón al señalar que, mientras menos sujetos migren hacia la informalidad, existen más posibilidades de control y, en consecuencia, de detectar eventuales operaciones sospechosas de LA/FT. Ello, especialmente, cuando se trata de desarrollar actividades de inteligencia financiera, a través del cotejo de los antecedentes que poseen las entidades financieras en Chile y/o en el extranjero, para lo cual la UAF requiere contar con la mayor cantidad de información posible acerca de las operaciones realizadas por los clientes bancarios. También es cierto que el de-risking puede llegar a potenciar la creación de industrias paralelas a la bancaria, las cuales no están sujetas a las mismas exigencias en materia de LA/FT que tienen los bancos, como ocurre, por ejemplo, con los operadores de criptomonedas. En estos supuestos, será mucho más difícil acceder a aquella información que permita detectar eventuales operaciones sospechosas de LA/FT.

En cualquier caso, la respuesta a este problema no parece consistir en obligar a los bancos a hacerse cargo de todos los riesgos de LA/FT, ya que ello podría conllevar un aumento excesivo en los costos derivados de la aplicación de las medidas de prevención. Probablemente, dichos costos serán trasladados luego a los clientes, de modo que estos podrían terminar migrando igualmente hacia la informalidad dificultándose, entonces, la aplicación de los controles. En consecuencia, para abordar adecuadamente esta problemática se considera necesario analizar, de manera particular, la situación de cada una de las categorías de clientes que pueden verse enfrentadas a la práctica del de-risking y plantear soluciones que se adapten a su realidad, en relación con los costos que los bancos han de asumir en estos supuestos.

Precisamente, a continuación, se identificarán las categorías de clientes que, en mayor medida, han sido vinculadas a este fenómeno, tanto en el extranjero como en nuestro país. El examen de su situación busca confirmar la idea que el de-risking encuentra su origen en el EBR, y su principal causa, en los costos que involucra la implementación de las medidas

37 GAFI (2014) y GAFI (2015). 
DDC. También se examinan algunas soluciones formuladas para afrontar dicha práctica y, según el caso, se proponen otras, a partir de la realidad de cada una de las señaladas categorías, bajo el entendido de que no existe una única solución posible a este respecto.

\section{CLIENTES AFECTADOS POR EL DE -RISKING A NIVEL INTERNACIONAL}

De acuerdo con lo evidenciado por organismos como el GAFI y el Banco Mundial, diversas entidades de crédito han decidido terminar unilateralmente sus relaciones de corresponsalía con bancos ubicados en países que no cuentan con una normativa de prevención del LA/FT suficientemente robusta, comparada con la que ellos aplican. Los bancos corresponsales han argumentado que los costos que generan estos vínculos no compensan las utilidades que producen, esencialmente en razón de los recursos que deben invertir en auditar los sistemas y procesos de las entidades que representan ${ }^{38}$.

Se ha señalado que esta práctica puede llegar a generar distorsiones a la libre competencia, a través de la concentración del mercado de la banca corresponsal, contribuyendo, además, a incrementar el costo de las transacciones y dificultando el acceso de los clientes de las entidades de crédito a las operaciones de comercio exterior ${ }^{39}$. Para afrontar este fenómeno, el GAFI ha elaborado guías especialmente diseñadas para la aplicación de las medidas de DDC en la banca de corresponsales, estructuradas a partir del EBR ${ }^{40}$. Por su parte, los bancos han implementado repositorios de información acerca de las entidades de crédito que operan internacionalmente. Funcionan con plantillas, a las cuales los bancos pueden acceder, para modificar y actualizar los datos relativos a los modelos de prevención de LA/FT que poseen, de modo de facilitar la aplicación de las medidas de DDC en estos $\operatorname{casos}^{41}$.

Otros afectados por la práctica del de-risking desarrollada por los bancos han sido las organizaciones sin fines de lucro y los servicios de transferencia de dinero, considerados de mayor riesgo por identificarse como potenciales vehículos para la financiación del terrorismo, particularmente, a partir de los atentados perpetrados el año 2001 en Estados Unidos. En este ámbito, se ha detectado que el de-risking suele darse cuando estas entidades operan con clientes ubicados en países que poseen una regulación débil en materia de prevención del LA/FT, que son objeto de sanciones internacionales, con gobiernos inestables, o derechamente, en situación de conflicto político ${ }^{42}$. El GAFI también ha elaborado guías acerca de la aplicación de las medidas de DDC respecto de las organizaciones sin fines de lucro y los servicios de transferencia de dinero, en el marco del EBR ${ }^{43}$.

38 GAFI (2016) p. 4 y World Bank Group (2015a).

39 Fuji-Rajani (2017) p. 220; International Chamber of Commerce (2016) p. 64; y, Lewis (2016).

40 Véanse GAFI (2016) y GAFI (2014a).

41 Son ejemplos de este tipo de bases de datos: Bankers Almanac, Depository Trust \& Clearing Cooperation (DTCC)-Clarient Entity Hub, Markit-Genpact, SWIFT KYC Registry y Thomson Reuters Accelus. Sin embargo, según se indica en Nadal (2016) pp. 30-32 y en Committee on PAYMENTS AND MARKet INFRAStruCtures (2016) pp. 19-22, dichas bases de datos presentan inconvenientes relativos a la fiabilidad de la información en razón de la heterogeneidad en la estructura de las plantillas.

42 Lewis (2016); GAFI (2014b) p. 1; Neumann (2017) p. 95; Fujil-Rajani (2017) p. 213; De Koker y otros (2017) pp. 137-138; World Bank Group (2015); Howard (2017) p. 75; y Durner y Shetret (2015) p. 8.

$43 \mathrm{Al}$ respecto, véanse GAFI (2015c) y GAFI (2016). 
Al formular estas guías, dicho organismo internacional ha señalado que en ningún caso es necesario que los bancos conozcan a los clientes de sus clientes (know your customer's customer o KYCC), ni siquiera en supuestos de mayor riesgo de LA/FT ${ }^{44}$. Esta aclaración resulta especialmente importante al momento de afrontar la aplicación de las medidas de DDC respecto de las entidades representadas, en el marco de las relaciones de corresponsalía bancaria y de las empresas que prestan servicios de transferencia de dinero, ya que en estos supuestos se suelen utilizar las cuentas corrientes que mantienen dichos sujetos en los bancos para gestionar aquellas operaciones requeridas por sus clientes.

A nivel internacional, también se ha observado de-risking respecto de quienes no cuentan con documentación de identidad u otros antecedentes fácilmente comprobables, como suele suceder, por ejemplo, con inmigrantes y refugiados. Los bancos han desarrollado esta práctica afirmándose en que no deben iniciar relaciones comerciales con clientes a los que no pueden aplicarse las medidas de $\mathrm{DDC}^{45}$. Esta manifestación del de-risking, relacionada directamente con la inclusión financiera ${ }^{46}$, ha sido afrontada por el GAFI sobre la base del enfoque basado en el riesgo, sosteniendo que es razonable considerar a ciertos clientes como de menor riesgo de LA/FT, en razón de los bajos montos o del carácter esporádico de las operaciones que realizan. De este modo, quienes están excluidos del sistema, podrían beneficiarse de la aplicación de medidas de DDC simplificadas, que son aquellas que se recomienda adoptar en los supuestos de menor riesgo de LA/FT ${ }^{47}$. Otra alternativa señalada por dicho organismo internacional para afrontar el desafío de la inclusión financiera por parte de los bancos -en el marco de una adecuada prevención del LA/FT- consiste en proveer de productos y servicios bancarios básicos a los sectores más vulnerables de la población, con un uso restringido y aplicando medidas de DDC de manera escalonada, a partir de ciertos límites de frecuencia y cantidad, previamente formulados para las transacciones que se pueden efectuar. En este punto, y tal como lo refiere el propio GAFI, en Chile tenemos camino avanzado, ya que contamos con las denominadas "cuentasRUT",

${ }^{44}$ GAFI (2015b) y GAFI (2016) p .4.

45 GAFI (2017) p. 39; GAFI (2013a) p. 28; y, GAFI (2012) p. 15.

46 La inclusión financiera se concibe en OECD/INFE (2012) p. 7 como el proceso de promoción de un acceso oportuno y adecuado a una amplia gama de servicios y productos financieros regulados. En el caso de Chile, la SBIF (2016) p. 32, señala que “...el 98\% de la población presenta algún tipo de inclusión financiera, de éstas, el $58 \%$ evidencia un tipo de inclusión financiera superior, es decir posee al menos algún producto con requerimiento de ingreso. El 40\% restante evidencia un tipo de inclusión básica...”. Luego, solo el 2\% de la población estaría excluida financieramente. Sin embargo, Cuevas (2016) sostiene que, no obstante el crecimiento experimentado respecto de la tenencia de productos financieros en nuestro país, un porcentaje importante de la población no estaría haciendo uso de ellos.

47 GAFI (2013) p. 33; AAMO (2012); y GAFI (2017) pp. 10 y 39. En este sentido, en GAFI (2017) pp. 11-12, se relata la situación de países como Estados Unidos, Canadá y Suiza, en que se permiten otros medios de identificación de los clientes, ampliando lo que se entiende como documentación válida (por ejemplo, tarjeta de votación, documentos tributarios, referencia de alquien que conozca al cliente que confirme su identidad). En Chile no se regula en detalle la adopción de medidas de DDC simplificadas, aun cuando el apartado II.2. del Capítulo 1-14 de la RAN CMF señala que: “...considerando la naturaleza, características y nivel de riesgo de los productos y servicios que contraten con este [cliente], podrán omitirse ciertos requisitos como, por ejemplo, la exigencia de una fotografía del cliente y la impresión digital. Las políticas deberán referirse a los procedimientos que deben aplicarse en estos casos”. La palabra entre paréntesis es mía. 
Toso Milos, Ángela "De-risking: una consecuencia indeseada del enfoque basado en el riesgo ..."

que están orientadas precisamente hacia la inclusión financiera, con límites establecidos para las operaciones realizadas, lo que permite un mayor control de los riesgos de LA/FT, al tiempo que conlleva una disminución de los costos derivados de la aplicación de medidas y controles en esta materia ${ }^{48}$.

Los clientes a los que se ha aplicado el de-risking a nivel internacional comprenden sectores muy diversos, aunque todos ellos poseen una característica en común, dada por el mayor riesgo de LA/FT que representan para los bancos, a la luz de lo establecido por el GAFI. Esta circunstancia permite reafirmar la idea de que esta práctica surge en el contexto del EBR. Respecto de los bancos representados y de los servicios de transferencia de dinero, el mayor riesgo está asociado a los clientes de dichos sujetos, especialmente cuando estos últimos no están sometidos a las mismas exigencias de prevención del LA/FT que el banco con el que pretenden mantener una relación de negocio. Por su parte, también se aprecia un riesgo importante en la contratación con clientes que presentan dificultades para ser identificados. En todos los supuestos señalados parece que los bancos deben incurrir en mayores costos si pretenden mitigar adecuadamente los riesgos de LA/FT, a la luz de las exigencias derivadas de la prevención de estos delitos, lo que permitiría explicar en buena medida las decisiones que han tomado ciertas entidades de crédito de desvincular a estas categorías de clientes.

\section{DE-RISKING EN NUESTRO PAÍS}

En Chile no hemos estado ajenos a la práctica del de-risking ni a controversias derivadas de esta. Prueba de ello es la denuncia recibida por la Fiscalía Nacional Económica (FNE) el año 2015, referida a la negativa, por parte de diversas instituciones bancarias, de apertura de cuentas corrientes a una casa de cambio. Según la denunciante, esta conducta de los bancos habría tenido por objeto restringir la competencia en el mercado de las operaciones de cambio de divisas. Por su parte, las entidades de crédito señalaron que su negativa obedeció a una decisión tomada en función de la falta de regulación y fiscalización suficiente de este tipo de actividades en Chile, de los costos de monitoreo de los riesgos de LA/FT que implica el mantenimiento de estas relaciones comerciales, y del consiguiente riesgo reputacional ${ }^{49}$. Como consecuencia de la denuncia presentada, la FNE inició una investigación, a partir de la cual constató "una cierta falta de transparencia" en las políticas de apertura y/o cierre de cuentas corrientes a casas de cambio y remesadoras de dinero, en términos tales que fue imposible determinar si la negativa de apertura y/o cierre de las mismas obedecía a un riesgo de LA/FT o si existía un ánimo anticompetitivo por parte de los bancos. En definitiva, tras la investigación realizada $-y$ como presupuesto para el archivo de la misma- los bancos accedieron a ajustar sus políticas internas de prevención del LA/FT, estableciendo, como requisito para la apertura de cuentas por parte de las casas de cambio

${ }^{48}$ GAFI (2017) pp. 7-9 y 13. Estos límites pueden revisarse en http://www.bancoestado.cl/imagenes/CuentaRUT/contratoCtaRUT.pdf. Fecha de consulta: 12.4.2019. Este tema es abordado extensamente en DURNER y SHetret (2015), así como en STANLEY y BuCKLey (2016). También existen diversos organismos internacionales que se han ocupado de esta materia. A modo de ejemplo, pueden citarse World bank Group (2017) y OECD (2013).

49 Resolución de la FNE, de 27 de junio de 2017, Rol No 2355-15. 
y remesadoras de dinero, que estas han de demostrar que cumplen con lo señalado en la Ley No 19.913 y en las circulares de la UAF, debiendo probarse tal circunstancia mediante una auditoría realizada por una empresa independiente y acreditada. Sin embargo, la FNE ha reconocido que ello no soluciona el problema de fondo, que vendría dado por la insuficiente regulación y fiscalización de los servicios ofrecidos por las casas de cambio y remesadoras de dinero en nuestro país, factor que habría determinado el de-risking en este caso ${ }^{50}$. Durante 2018, se presentaron nuevas denuncias a este respecto, las cuales fueron las cuales fueron desestimadas ${ }^{51}$.

Antes que la investigación de la FNE tuviera lugar, una conocida casa de cambio y remesadora de dinero demandó a su banco ante el TDLC, en virtud de la decisión de esta entidad de crédito de cerrar las cuentas que mantenía dicha casa de cambio con ella ${ }^{52}$. El banco indicó que estas cuentas le significaban una carga adicional, consistente en "...un control exhaustivo por parte de los profesionales y técnicos que laboran en la División Global de Cumplimiento, como lo ordena la normativa de prevención del lavado de activos..."; y que, por tanto, no eran rentables ${ }^{53}$. Finalmente, la demanda fue rechazada por el tribunal, en atención, entre otros factores, al hecho que la casa de cambio podría utilizar los servicios de bancos distintos al demandado, de modo que el cierre de sus cuentas no habría significado su exclusión de los mercados en que opera, no evidenciándose conducta anticompetitiva alguna en este caso $^{54}$. Si bien esta sentencia se refiere a una desvinculación individual -y no a una realizada "en bloque" por diversas instituciones bancarias-, parece importante traerla a colación aquí. Primeramente, porque llama la atención que la entidad de crédito, para tomar la decisión de desvincular al cliente, indicó que el cierre de las cuentas se justificaba porque la relación comercial con la casa de cambio no resultaba rentable, revelando que este parece ser el criterio predominante al momento de optar por el de-risking. En segundo lugar, porque este caso podría concebirse como un antecedente del de-risking que se revelaría después con relación a las casas de cambio y remesadoras de dinero, a través de la investigación efectuada por la FNE. Por último, porque esta sentencia, conjuntamente con el expediente llevado adelante por la FNE, evidencia las dificultades que se presentan para considerar, tanto las desvinculaciones individuales como aquellas realizadas "en bloque", en sí mismas, como atentatorias a la libre competencia". Esta última circunstancia da cuenta del hecho que las instancias a las que acudieron las casas de cambio y remesadoras de dinero en estos casos no parecen adecuadas ni suficientes para evitar el de-risking, en tanto fenómeno que surge como una consecuencia (indeseada) de la aplica-

\footnotetext{
50 Informe, de 27 de junio de 2017, en Rol No 2355-15, No 26, 35-36, $42-43$ y 48.

51 Resolución de la FNE, de 4 de junio de 2018 e informe de 1 junio de 2018, en Rol No 2492-18.

52 En particular, por haber cometido con ello prácticas exclusorias; explotación abusiva de poder de mercado; negativa de venta; y, competencia desleal. Al respecto, véase Afex Transferencias y Cambios Ltda. y OTRO contra Banco de Chile (2013), vistos, punto 1.17.

53 Afex Transferencias y Cambios Ltda. y otro contra Banco de Chile (2013), vistos, puntos 4.4 y 4.5.

54 Afex Transferencias y Cambios Ltda. y otro contra Banco de Chile (2013), considerandos trigésimo octavo, quincuagésimo cuarto y quincuagésimo noveno.

55 Esta dificultad también se observa en sentencias extranjeras como Dahabshill Transfer Services Ltd. V. Barclays Bank Plc (2013), así como los demás casos tratados en De KoKer y otros (2017) p. 140.
} 
Toso MiLos, Ángela "De-risking: una consecuencia indeseada del enfoque basado en el riesgo ..."

ción del EBR, en el marco del sistema de prevención del LA/FT. Ello, entre otras razones, porque se trata de procedimientos cuyo principal objeto es evitar o subsanar conductas anticompetitivas, de modo que no tienen por finalidad prevenir estos delitos.

Otro supuesto reciente de de-risking es el que que ha afectado a los operadores de criptomonedas, quienes han iniciado diversos procedimientos ante el TDLC por este motivo $^{56}$. En estos procesos, los bancos han señalado que su decisión -de no abrir o bien de cerrar cuentas- estaría basada en el riesgo del LA/FT que dichos sujetos representan, en el marco de la aplicación de las medidas de DDC. Apoyándose en lo indicado por el GAFI, sostienen que las operaciones con criptomonedas hacen muy difícil la implementación de este tipo de medidas, por cuanto favorecerían el anonimato de quienes las llevan a cabo y dificultarían el acceso expedito a los registros de las transacciones ${ }^{57}$. Sostienen, en consecuencia, que se encuentran plenamente facultados para denegar la apertura o decretar el cierre de las cuentas en estos casos. Finalmente, los bancos han puesto de relieve el hecho que los operadores de criptomonedas realizan una actividad no regulada en Chile, y que -a diferencia de lo que ocurre con las casas de cambio y las remesadoras de dinero- no están obligados a contar con modelos de prevención del LA/FT, factor que contribuye a incrementar los riesgos de vincularse comercialmente con ellos. Por su parte, los operadores de criptomonedas han señalado que no es efectivo que las transacciones en las que intermedian favorecen el anonimato, ya que la tecnología blockchain permite identificar a sus participantes. Además, argumentan que cuentan con modelos voluntarios de prevención del LA/FT, que permitirían mitigar los riesgos en este tipo de operaciones. Estos procedimientos aún están en curso. Sin embargo, el TDLC ordenó, como medida cautelar en dichos expedientes, la celebración de nuevos contratos (de cuenta corriente o cuenta vista) entre los operadores de criptomonedas y los bancos demandados ${ }^{58}$.

En forma paralela al inicio de las causas ante el TDLC, los operadores de criptomonedas entablaron diversos recursos de protección, con la finalidad de que se ordenara a los bancos la reapertura de las cuentas que mantenían con ellos, señalando que el cierre de las mismas implicaba un atentado al derecho a la igualdad ante la ley, establecido en el artículo 19 No 2 de la Constitución Política de la República, además de vulnerar lo dispuesto en los artículos 19 No 21 y 26 de nuestra carta magna. En lo que dice relación con el fenómeno objeto de este trabajo, vale la pena destacar que las entidades bancarias sostuvieron que la decisión de cerrar las cuentas a los operadores de criptomonedas no constituye una medida discriminatoria ni arbitraria, por cuanto se trata de la desvinculación de una categoría íntegra de clientes a partir del hecho que su actividad no está regulada en Chile, esto añadido a los demás riesgos de LA/FT que dichos clientes representan, sin que haya existido ninguna diferencia de trato entre ellos. Este argumento sugiere que estamos en presencia de un su-

\footnotetext{
56 TDLC, Roles No C-349-2018, No C-350-2018 y C-354-2018.

57 GAFI (2014c) p. 9 y GAFI (2015d) p. 11. Acerca de los riesgos de LA/FT generados a partir del empleo de criptomonedas, véase también BEDECARRATZ (2018) pp. 90-95.

58 Estos argumentos y actuaciones procesales constan en los procesos ventilados ante el TDLC, individualizados en la nota No 56 de este trabajo.
} 
puesto arquetípico de de-risking, incorporado expresamente como tal en los manuales de prevención LA/FT de ciertas instituciones bancarias ${ }^{59}$.

En estos casos -como suele suceder en la mayoría de los supuestos de desvinculación por parte de los bancos asociada a la prevención del LA/FT-, nuestros tribunales superiores, a la luz de lo dispuesto en el artículo 1.545 del Código Civil y en el Capítulo 2-2 de la RAN $\mathrm{CMF}^{60}$, han reconocido la facultad que tienen dichas entidades de crédito para poner término de manera unilateral al contrato de cuenta corriente, en la medida que se cumpla con lo señalado en el mismo y en el artículo 17 B, letra b), de la Ley 19.496, sobre protección de los derechos de los consumidores, cuando corresponda. Ello supone la inclusión en el contrato de las causas que permiten a un prestador de servicios financieros terminar unilateralmente esta relación de negocios ${ }^{61}$.

Según se puede observar, al igual como ha ocurrido en el extranjero, en nuestro país el de-risking ha operado respecto de categorías de clientes de mayor riesgo de LA/FT, a la luz de los estándares formulados por el GAFI, fundamentalmente debido a la falta de regulación y/o supervisión de la actividad que realizan ${ }^{62}$. Los bancos han reconocido que en estos supuestos los costos asociados a la aplicación de las medidas DDC en el marco del EBR se incrementan, haciendo poco rentable seguir manteniendo dichas relaciones de negocio.

En cuanto a las soluciones que pueden proponerse para afrontar el de-risking en estos casos, la formulación de una normativa adecuada que regule los diversos giros sensibles al LA/FT, y una fiscalización suficiente de la misma, pueden contribuir a evitar esta práctica. Sin embargo, esto no siempre será posible, considerando el rápido desarrollo del tráfico económico, que implica el surgimiento constante de nuevos modelos de negocios.

También puede resultar conveniente incorporar expresamente en la regulación nacional de prevención del LA/FT la aclaración realizada por el GAFI, en el sentido que los bancos no están obligados a "conocer a los clientes de sus clientes". Así se facilitaría la aplicación de las medidas DDC, evitando el de-risking en casos como el de los operadores de criptomonedas, a través de una disminución de los costos asociados al cumplimiento de la normativa de prevención del LA/FT.

Finalmente, al momento de afrontar el fenómeno del de-risking, no hay que olvidar la existencia de un factor determinante en su desarrollo, y es que, por regla general, los bancos pueden decidir con quién mantienen relaciones comerciales, derecho que ha sido

\footnotetext{
59 Véase Orionx SpA con Banco del Estado de Chile (2018), considerando quinto.

60 El apartado I.10. del capítulo 2-2 RAN CMF, señala: "La cuenta corriente podrá ser cerrada unilateralmente por el banco...".

61 En este sentido, pueden consultarse E.A.G.Y. CON BANCO SANTANDER-CHILE (2010); J.V.G.R. CON BANCO DE CRÉdito E INVERsiones (2014); J.A.L.F CON BANCO EDWARDS CitY (2014); V.L.C.M. CON BANCO ITAÚ (2016); INtervalores Corredores de Bolsa Limitada con Banco Bilbao Viscaya Argentaria Chile (2017); Cryptomkt Spa con Banco Scotiabank Chile S.A. (2018); Surbtc SpA con Banco Itaú Corpbanca (2018); y, OrionX SpA con BANCO DEL ESTADO DE CHILE (2018a). Esta tendencia también puede observarse en sentencias extranjeras, como es el caso de BREDENKAMP V. STANDARDBANK (2010) y HLONGWANEAND OTHERS V. ABSA BANK LIMITED AND ANOTHER (2016), ambas de Sudáfrica, así como en E-TRANSINTERNATIONAL FINANCE LTD V. KIWIBANK LTD (2016), pronunciada en Nueva Zelanda, todas analizadas en DE KoKer y otros (2017) pp. 136-140.

62 En GAFI (2014c) pp. 9-10, se identifica este riesgo dentro de aquellos vinculados a las operaciones con criptomonedas.
} 
reconocido expresamente y de forma reiterada por nuestros tribunales ${ }^{63}$. Con la finalidad de proteger a los clientes -especialmente en aquellos supuestos de de-risking injustificado-, conviene observar de cerca otras experiencias que pueden servir de orientación al momento de formular soluciones a esta problemática. Así, por ejemplo, en la Unión Europea, la Directiva 2014/92/EU, de 2014, sobre la comparabilidad de las comisiones conexas a las cuentas de pago, el traslado de cuentas de pago y el acceso a cuentas de pago básicas, reconoce el derecho de los consumidores de contar con servicios financieros, aunque no se trata de un derecho absoluto, ya que el banco se puede negar a abrir una cuenta, o decidir cerrarla, si el cliente incumple la normativa de prevención del LA/FT o abusa de su derecho, cometiendo fraude contra el banco ${ }^{64}$. Lo mismo ocurre en Canadá, a partir de lo dispuesto en su Bank $A c t^{65}$. Por último, y particularmente en relación con las remesadoras de dinero, la Directiva 2015/2366/EU, de 2015, sobre servicios de pago en el mercado interior, establece que los bancos deben proveer servicios sin obstáculos y de manera eficiente a esos clientes, de modo que, si una entidad de crédito rechaza prestar dichos servicios, debe entregar a las autoridades las razones que han motivado su negativa ${ }^{66}$.

\section{CONCLUSIONES}

1. En el marco del EBR, los bancos deben, en primer lugar, identificar y valorar las relaciones de negocios que establecen en función del riesgo de LA/FT que estas representan. Luego, a partir de dicha valoración, el régimen jurídico de prevención contempla la aplicación de medidas adicionales de DDC para gestionar y monitorear aquellos vínculos comerciales de mayor riesgo de LA/FT. Precisamente, al desempeñar estas tareas los bancos han tomado la decisión, en determinados casos, de evitar los riesgos de LA/FT en vez de gestionarlos, dando lugar al fenómeno del de-risking. El principal motivo que genera dicha práctica parece radicar en los costos económicos que implica la regulación de prevención del LA/FT, de tal manera que, cuando se opta por el de-risking, se estaría haciendo primar dichos costos frente a los menores beneficios que parece representar para los bancos el cumplimiento de las medidas de prevención de estos delitos.

2. En cuanto a las consecuencias atribuidas tradicionalmente al de-risking, el GAFI ha señalado que, cuanta más cobertura posea un sistema financiero, mayores son las posibilidades de prevenir eficazmente el LA/FT. Luego, sostiene que, si una persona (natural o jurídica) no tiene acceso al sistema, hay más probabilidades de que utilice canales financieros informales, con lo que se incrementaría el riesgo asociado a la comisión de estos delitos. Lo indicado por el GAFI evidencia la necesidad de reflexionar acerca de los verdaderos efectos que el de-risking puede provocar en materia de prevención del LA/FT, los cuales no coinciden necesariamente con los que indica dicho organismo. En todo caso, el GAFI tiene razón cuando

\footnotetext{
63 Véase la nota anterior.

${ }^{64}$ Artículo 16.4, Directiva 2014/92/EU, de 2014.

65 SC 1991, c 46, s 448.1(1).

66 Artículo 36, Directiva 2015/2366/EU, de 2015.
} 
señala que, mientras menos sujetos migren hacia la informalidad, existen más posibilidades de control y, en consecuencia, de detectar eventuales operaciones sospechosas de LA/FT.

3. Para afrontar adecuadamente el fenómeno del de-risking es necesario analizar de manera particular la situación de cada una de las categorías de clientes que pueden verse enfrentadas a esta práctica y plantear soluciones que se adapten a su realidad, en relación con los costos que los bancos han de asumir en estos supuestos. Entre las categorías de clientes a las que se les ha aplicado el de-risking en el extranjero, se cuentan los bancos representados; las organizaciones sin fines de lucro; los servicios de transferencia de dinero; y, los clientes que no cuentan con documentación de identidad u otros antecedentes fácilmente comprobables, como suele suceder con inmigrantes y refugiados. En Chile, las casas de cambio, las remesadoras de dinero y los operadores de criptomonedas han sido objeto de de-risking. En consecuencia, esta práctica ha afectado a sectores muy diversos, aunque todos ellos poseen una característica en común, dada por el mayor riesgo de LA/FT que representan para los bancos, a la luz de lo establecido por el GAFI, por lo que dichas entidades de crédito debieran aplicar medidas de DDC reforzadas a su respecto. En ese contexto, los bancos han considerado a estos clientes poco rentables. Esta circunstancia permite confirmar la idea de que esta práctica surge en el contexto del EBR, a propósito de la aplicación de las medidas de DDC, y que está basada esencialmente en los costos que conlleva para los bancos el cumplimiento de la normativa de prevención del LA/FT.

4. Dentro de las posibles soluciones que pueden formularse para afrontar el de-risking, particularmente respecto de aquellos clientes más vulnerables, se cuenta la provisión de productos y servicios bancarios básicos, con un uso restringido a partir de determinados límites de frecuencia y cantidad en las operaciones. Por otra parte, la incorporación expresa en la normativa de prevención del LA/FT de la aclaración realizada por el GAFI, en el sentido de que los bancos no están obligados a "conocer a los clientes de sus clientes", también puede ayudar a evitar esta práctica en el caso de las relaciones de corresponsalía, las organizaciones sin fines de lucro, las casas de cambio, las remesadoras de dinero y los operadores de criptomonedas. Respecto de estas tres últimas categorías de clientes, la formulación de una normativa adecuada en materia de LA/FT y una fiscalización suficiente respecto de su cumplimiento también pueden contribuir a prevenir el de-risking. Por último, al momento de plantear soluciones a esta problemática, no hay que olvidar que, por regla general, los bancos tienen la facultad de decidir con quién mantienen relaciones comerciales. A fin de proteger a los clientes, especialmente frente a supuestos de de-risking injustificado, conviene reflexionar en torno a la posibilidad de elaborar normativa, como la europea y canadiense, que reconozca expresamente, y regule en detalle, el derecho de los consumidores a contar con servicios financieros y que obligue a las entidades de crédito a justificar su negativa a prestarlos, cuando corresponda. 


\section{BIBLIOGRAFÍA CITADA}

AAмо, Bjørn (2012): "Promoting Financial Inclusion through Proportionate Standards and Guidance”. Disponible en: http://www.fatf-gafi.org/publications/fatfgeneral/documents/fatfs focusonfinancialinclusionprotectingtheintegrityoftheglobalfinancialsystem. html. Fecha de consulta: 2 de abril de 2019.

Alarcón, Antonio (2016): Manual Teórico-Práctico del Compliance Officer (Madrid, Sepin). Artaza, Osvaldo (2013): "Sistemas de prevención de delitos o programas de cumplimiento. Breve descripción de las reglas técnicas de gestión del riesgo empresarial y su utilidad en sede jurídico penal”, Política Criminal, vol. 8, No 16: pp. 544-573.

Asociación de Supervisores Bancarios de las Américas (ASBA) (2017): "Una visión general sobre el de-risking: causas, efectos y soluciones". Disponible en: http://www.asbasupervision.com/es/bibl/i-publicaciones-asba/i-2-otros-reportes/1597-una-vision-general-sobre-el-de-risking-causas-efectos-y-soluciones/file. Fecha de consulta: 2 de abril de 2019.

Ayala de la Torre, José María (2016): Compliance. Claves Prácticas (Madrid, Francis Lefebvre).

Basel Commitee on Banking Supervision (2016): Sound Management of risk related to money laundering and financing of terrorism. Disponible en: https://www.bis.org/bcbs/ publ /d353.pdf. Fecha de consulta: 2 de abril de 2019.

Bedecarratz, Francisco (2018): "Riesgos delictivos de las monedas virtuales: Nuevos desafíos para el derecho penal”, Revista Chilena de Derecho y Tecnología, vol. 7, No 1: pp. 79-105.

BRUNEEN, Francisco (2017): "La prevención del fraude y de la corrupción”, en PuYOL, Javier (dir.), Guía para la implantación del Compliance en la empresa (Barcelona, Bosch-Wolters Kluwer) pp. 175-208.

Carbayo, Francisco (2016): “Abogados, Riesgos Legales y Compliance”, Diario La Ley, No 8702, Ref. D-68.

Carrau, Rafael (2016): Compliance para Pymes (Valencia, Tirant lo Blanch).

Coca, Ivó (2013): “¿Programas de Cumplimiento Normativo como forma de autorregulación regulada?”, en Silva, Jesús María (dir.) y Montaner, Raquel (coord.), Criminalidad de empresa y Compliance. Prevención y reacciones corporativas (Barcelona, Atelier) pp. 43-76.

Comité de Supervisión Bancaria de Basilea (2001): "Debida diligencia con la clientela de los bancos”. Disponible en: www.bis.org. Fecha de consulta: 2 de abril de 2019.

Committee on Payments and Market Infrastructures (2016): "Consultative Report. Correspondent Banking”. Disponible en: www.bis.org. Fecha de consulta: 2 de abril de 2019.

Cuevas, Cristián (2016): "Una nueva mirada a la inclusión financiera en Chile”, Estudios Públicos, vol. 143: pp. 77-107.

Del Cid, Juan Miguel (2007): Blanqueo internacional de capitales. Cómo detectarlo y prevenirlo (Barcelona, Deusto). 
De Koker, Louis; Singh, Supriya; y, Capal, Jonathan (2017): "Closure of Bank Accounts of Remittance Service Providers: Global Challenges and Community Perspectives in Australia”, University of Queensland Law Journal, vol. 36, No 1: pp. 119-154.

Durner, Tracey y SHETRET, Liat (2015): "Bank de-risking and its effects on financial Inclusion. An exploratory study". Disponible en: https:/www.oxfam.org/sites/www.oxfam. org/files/file_attachments/rr-bank-de-risking-181115-en_0.pdf. Fecha de consulta: 2 de abril de 2019.

Enseñat, Sylvia (2016): Manual del Compliance Officer (Cizur Menor, Thomson ReutersAranzadi).

Fujil-Rajani, Riki (2017): "Money Remitters Left out in the Cold: Blanket De-Risking Policies, Counterterrorism and Government Intervention in New Zealand", Auckland University Law Review, vol. 23: pp. 204-234.

Geiger, Hans y Wuensch, Oliver (2006): “The Fight Against Money Laundering-An Economic Analysis of a Cost-Benefit Paradoxon”. Disponible en: http://www.hansgeiger.ch/ files/ 060929_AmlEconomicAnalysis.pdf. Fecha de consulta: 2 de abril de 2019.

Greenberg, Theodore y otros (2009): "Stolen Asset Recovery. Politically Exposed Persons. A Policy Paper on Strengthening Preventive Measures". Disponible en: http:// siteresources.worldbank.org /EXTSARI/Resources/5570284-1257172052492/PEPs-ful. pdf?resourceurlname=PEPs-ful.pdf. Fecha de consulta: 2 de abril de 2019.

Gómez, Iñigo (2017): "Diseño de un sistema de Compliance”, en Puyol, Javier, Guía para la implantación del Compliance en la empresa (Barcelona, Bosch-Wolters Kluwer) pp. 175-208.

Grupo de Acción Financiera Internacional (2017): "Anti-Money Laundering and Terrorist Financing measures and Financial Inclusion. With a Supplement on Customer Due Diligence". Disponible en: http:/www.fatf-gafi.org/media/fatf/content/images/ Updated-2017-FATF-2013-Guidance.pdf. Fecha de consulta: 2 de abril de 2019.

Grupo de Acción Financiera Internacional (2016): "FATF Guidance on Correspondent Banking Services”. Disponible en: http://www.fatf-gafi.org/media/fatf/documents/ reports/Guidance-Correspondent-Banking-Services.pdf. Fecha de consulta: 2 de abril de 2019.

Grupo de Acción Financiera Internacional (2016a): "Guidance on a Risk Based Approach for Money or Value Transfer Services”. Disponible en: http://www.fatf-gafi.org/ media/fatf/documents/reports/Guidance-RBA-money-value-transfer-services.pdf. Fecha de consulta: 2 de abril de 2019.

Grupo de Acción Financiera Internacional (2015): "FATF takes action to tackle derisking”. Disponible en: http://www.fatf-gafi.org/publications/fatfrecommendations/ documents/fatf-action-to-tackle-de-risking.html. Fecha de consulta: 2 de abril de 2019.

Grupo de Acción Financiera Internacional (2015a): "Drivers for "de-risking” come beyond anti-money laundering/terrorist financing”. Disponible en: http://www.fatf-gafi. org/doc uments/news/derisking-goes-beyond-amlcft.html. Fecha de consulta: 2 de abril de 2019.

Grupo de Acción Financiera Internacional (2015b): "Dialogue with the Private Sector". Disponible en http:/www.fatf-gafi.org/documents/news/private-sector-forummarch-2015.html. Fecha de consulta: 2 de abril de 2019. 
Toso Milos, Ángela "De-risking: una consecuencia indeseada del enfoque basado en el riesgo ...”

Grupo de Acción Financiera Internacional (2015c): "Best Practices Paper on Combating the Abuse of Non-Profit Organizations”. Disponible en: http://www.fatf-gafi.org/ publications/fatf recommenda tions/documents/bpp-combating-abuse-npo.html. Fecha de consulta: 2 de abril de 2019.

Grupo de Acción Financiera Internacional (2015d): "Directrices para un enfoque basado en riesgo". Disponible en: https://www.uaf.cl/asuntos/docs_gafi.aspx?id=3. Fecha de consulta: 2 de abril de 2019 .

Grupo de Acción Financiera Internacional (2014): "FATF clarifies risk-based approach: case by case, not wholesale de-risking". Disponible en: http://www.fatf-gafi.org/publications/fatf general/documents/rba-and-de-risking.html. Fecha de consulta: 2 de abril de 2019.

Grupo de Acción Financiera Internacional (2014a): "Guidance for a Risk-Based Approach. The Banking Sector”. Disponible en: http://www.fatf-gafi.org/media/fatf/documents/reports/Risk-Based-Approach-Banking-Sector.pdf. Fecha de consulta: 2 de abril de 2019.

Grupo de Acción Financiera Internacional (2014b): "Risk of Terrorist Abuse in NonProfit Organisations". Disponible en: http://www.fatf-gafi.org/media/fatf/documents/ reports/Risk-of-terrorist-abuse-in-non-profit-organisations.pdf. Fecha de consulta: 2 de abril de 2019.

Grupo de Acción Financiera Internacional (2014c): "Informe del GAFI. Monedas Virtuales. Definiciones Claves y Riesgos Potenciales de LA/FT”. Disponible en: https:// www.uaf.cl/asuntos/descargar.aspx?arid=961. Fecha de consulta: 2 de abril de 2019.

Grupo de Acción Financiera Internacional (2013a): "Money Laundering and Terrorist Financing Measures and Financial Inclusion”. Disponible en: http://www.fatf-gafi.org/ media/fatf/documents/reports/AML_CFT_Measures_and_Financial_Inclusion_2013. pdf. Fecha de consulta: 2 de abril de 2019.

Grupo de Acción Financiera Internacional (2012): "Estándares internacionales sobre la lucha contra el lavado de activos, el financiamiento del terrorismo y el financiamiento de la proliferación de armas de destrucción masiva. Las Recomendaciones del GAFI". Disponible en: http://www.gafilat.org/UserFiles/documentos/es/Las_Nuevas_40_Recomendaciones.pdf. Fecha de consulta: 2 de abril de 2019.

Grupo de Acción Financiera Internacional (2007): "Guidance on the Risk-Based Approach to Combating Money Laundering and Terrorist Financing. High Level Principles and Procedures". Disponible en: http://www.fatf-gafi.org/media/fatf/documents/ reports/High\%Level\%20Principles\%20and\%20Procedures.pdf. Fecha de consulta: 2 de abril de 2019.

Howard, Cory (2017): "Financial Crime Compliance Self- Governance: Applying the Faragher Defense to Bank Secrecy Act/Anti-Money Laundering Violations", The University of Memphis Law Review, vol. 48: pp. 45-82.

Howard, Cory (2017a): "Warding off Scylla and Charybdis: The Conflicts Financial Institutions face in the Context of BSA/AML and Fair Lending Responsibilities", Business \& Bankruptcy Law Journal, vol. 207: pp. 207-220. 
Biblioteca de Congreso Nacional (2019): "Historia de la ley 21.130, moderniza la legislación bancaria”. Disponible en: https://www.bcn.cl/historiadelaley/historia-de-la-ley/ vista-expandida/7613/ Fecha de consulta: 19 de agosto de 2019.

International Chamber of Commerce (2016): "Rethinking Trade \& Finance. ICC Global Trade Finance Survey Report 2016”. Disponible en: http://store.iccwbo.org/ content/uploaded/ pdf/ICC_Global_Trade_and_Finance_Survey_2016.pdf. Fecha de consulta: 2 de abril de 2019.

LEWIS, Daniel (2016): "Keynote speech ESAAMLG Public/Private Sector Dialogue". Disponible en: http://www.fatf-gafi.org/publications/fatfgeneral/documents/keynote-esaamlg-public-private-sector.html. Fecha de consulta: 2 de abril de 2019.

LÓpez, Óscar (2017): "Gestión del riesgo de Compliance y su control", en PuYOL, Javier, Guía para la implantación del Compliance en la empresa (Barcelona, Bosch-Wolters Kluwer, Barcelona) pp. 235-253.

Marneffe, Wim y Vereeck, Lode (2011): "The meaning of regulatory costs", European Journal of Law and Economics, vol. 32, No 3: pp. 341-356.

Mariño, Gladys; Chaparro, Fidela; y, Sandoval, Inelia (2014): "Determinantes en la prevención del riesgo para el lavado de activos y la financiación del terrorismo (LA/FT) en el sector real", Ad-Minister, No 25: pp. 7-35.

NADAL, Emilio (2016): "Banca de corresponsalía, de-risking y blanqueo de capitales". Disponible en: https://repositorio.comillas.edu/xmlui/bitstream/handle/11531/15574/ TFM000567.pdf?sequence $=1$. Fecha de consulta: 2 de abril de 2019 .

Neumann, Peter (2017): "Don't Follow the Money: The Problem with the War of Terrorist Financing", Foreign Affairs, vol. 96: pp. 93-102.

NeIra, Ana María (2016): "La efectividad de los criminal compliance programs como objeto de prueba en el proceso penal", Política Criminal, vol. 11, No 22: pp. 467-520.

Nieto, Adán (2015): "El Cumplimiento Noramativo", en Nieto, Adán y otros (2015): Manual de Cumplimiento Penal en la Empresa (Valencia, Tirant lo Blanch) pp. 25-48.

Organization for Economic Co-operation and Development (OECD) (2014): "OECD Regulatory Compliance Cost Assessment Guidance”. Disponible en: https://www. normenkontrollrat.bund.de/resource/blob/244032/444040/bb5c3d481212a08f42ccd07e0edc471e/oecd-regulatory-compliance-cost-guidance-data.pdf. Fecha de consulta: 2 de abril de 2019.

Organization for Economic Co-operation and Development (OECD) (2013): "Financial Literacy and Inclusion. Results of OECD /INFE Survey Across Countries and by Gender". Disponible en: https://www.oecd.org/daf/fin/financial-education/TrustFund2013_OECD_INFE_Fin_Lit_and_Incl_SurveyResults_by_Country_and_Gender.pdf. Fecha de consulta: 2 de abril de 2019.

Organization for Economic Co-operation and Development (OECD) y international Gateway for Financial Education (INFE) (2012): "High-level principles on national strategies for financial education". Disponible en: http://www.oecd.org/daf/fin/ financial-education/OECD_INFE_High_Level_Principles_National_Strategies_Financial_Education_APEC.pdf. Fecha de consulta: 2 de abril de 2019. 
Olaya, Cristina y Sánchez, Ana Luisa (2017): Compliance. Guía Práctica de identificación, análisis y evaluación de riesgos (Cizur Menor, Thomson Aranzadi).

PiñA, Juan Ignacio (2012): Modelos de prevención de delitos en la empresa (Santiago, Thomson Reuters-Abeledo Perrot).

Ross, Stuart y Hannan, Michelle (2007): "Money laundering regulation and risk-based decision making", Journal of Money Laundering Control, vol. 10, № 1: pp. 106-115.

SÁncheZ, Emilio (2006): "Estrategias de investigación en los delitos económicos complejos. La criminalidad económica organizada”, en Gimeno, Miguel Ángel, Derecho penal económico. Estudios de Derecho judicial (Madrid, Consejo General del Poder Judicial) pp. 11-48.

SÁncheZ, Emilio (2002): "Blanqueo de capitales en el sector bancario y corrupción. Aspectos prácticos", en FERRÉ, Juan Carlos, Blanqueo de dinero y corrupción en el sistema bancario. Delitos financieros, fraude y corrupción en Europa, vol. II (Salamanca, Ediciones Universidad de Salamanca) pp. 305-342.

Saperstein, Lanier; Sant, Geoffrey; y NG, Michelle (2015): "The Failure of Anti-Money Laundering Regulation: Where is the Cost-Benefit Analysis”. Disponible en: https:// scholarship.law.nd.edu/cgi/viewcontent.cgi?article=1015\&context=ndlr_online. Fecha de consulta: 2 de abril de 2019.

Sepblac (2007): "Guía para la prevención del riesgo de blanqueo de capitales en banca de corresponsales”. Disponible en www.sepblac.es. Fecha de consulta: 2 de abril de 2019.

Stanley, Rebecca y Buckley, Ross (2016): "Protecting the West, excluding the rest: the impact of the AML/CFT regime on financial inclusion in the Pacific and potential responses", Melbourne Journal of International Law, vol. 17, № 1: pp. 1-22.

Superintendencia de Bancos e Instituciones Financieras (SBIF) (2016): "Informe de Inclusión Financiera”. Disponible en: https://www.sbif.cl/sbifweb3/internet/archivos/ publicacion_11243.pdf. Fecha de consulta: 2 de abril de 2019.

ThOmson Reuters (2018): "Revealing de true cost of financial crime. 2018 survey report". Disponible en: https:/risk.thomsonreuters.com/content/dam/openweb/documents/ $\mathrm{pdf} /$ risk/report/true-cost-of-financial-crime-global-focus.pdf. Fecha de consulta: 2 de abril de 2019.

Toso, Angela (2014): "Prevención del lavado de activos y crédito documentario: ¿a quién debe conocer el banco emisor? Una respuesta desde el Derecho Privado", Revista de Derecho Universidad Católica del Norte, año 21, No 2: pp. 401-436.

Wilkins, Roger (2014): "The danger of driving both illicit markets and financial exclusion”. Disponible en: http://www.fatf-gafi.org/fr/publications/inclusionfinanciere/documents/danger-illicit-markets-financial-exclusion.html?hf=10\&b=0\&s=desc(fatf_releasedate). Fecha de consulta: 2 de abril de 2019.

World Bank Group (2017): "Financial Inclusion and Inclusive Growth. A review of Recent Empirical Evidence. Policy Research Working Paper No 8040”. Disponible en: http://documents. worldbank.org/curated/en/403611493134249446/pdf/WPS8040. pdf. Fecha de consulta: 2 de abril de 2019.

World Bank Group (2015): "Report on the G20 Survey on De-risking Activities in the Remittance Market. Working Paper No 101071”. Disponible en: http://documents. 
worldbank.org/curated/en/ 679881467993185572/Report-on-the-G20-survey-in-derisking-activities-in-the-remittance-market. Fecha de consulta: 2 de abril de 2019.

World Bank Group (2015a): "Withdrawal from Correspondent Banking; Where, Why, and What to Do About It. Working Paper No 101098”. Disponible en: http://documents.worldbank.org/curated/en/113021467990964789/pdf/101098-revised-PUBLIC-CBR-Report-November-2015.pdf. Fecha de consulta: 2 de abril de 2019.

\section{NORMATIVA CITADA}

LEY No 21.130 (12/01/2019), moderniza la legislación bancaria.

LEY No 21.000 (23/02/2017), crea la Comisión para el Mercado Financiero.

LEY No 20.393 (2/12/2009), sobre responsabilidad penal de las personas jurídicas.

LEY No 19.913 (18/12/2003), crea la Unidad de Análisis Financiero y modifica diversas disposiciones en materia de lavado y blanqueo de activos.

Decreto con Fuerza de Ley No 3 (19/12/1997), del Ministerio de Hacienda, que fija el texto refundido, sistematizado y concordado de la Ley General de Bancos.

Circular No 49, de la Unidad de Análisis Financiero (3/12/2012), ordenamiento y sistematización de las instrucciones de carácter general impartidas por la Unidad de Análisis Financiero a los sujetos obligados a informar.

Circular No 57, de la Unidad de Análisis Financiero (12/6/2017), imparte instrucciones sobre obligaciones de identificación, verificación y registro de datos de beneficiario(s) final(es) de personas y estructuras jurídicas.

Circular No 59, de la Unidad de Análisis Financiero (24/5/2019), introduce modificaciones a la Circular No 49, de 2012.

Capítulo 1-14 de la Recopilación Actualizada de Normas de la Comisión para el MerCADO FINANCIERO.

Directiva 2014/92/UE del Parlamento Europeo y del Consejo (DOUE L 257/214, 28/8/2014), sobre la comparabilidad de las comisiones conexas a las cuentas de pago, el traslado de cuentas de pago y el acceso a cuentas de pago básicas.

Directiva 2015/2366/UE del Parlamento Europeo y del Consejo (DOUE L 337/35, 23/12/2015), sobre servicios de pago en el mercado interior y por la que se modifican las Directivas 2002/65/CE, 2009/110/CE y 2013/36/UE y el Reglamento (UE) no 1093/2010 y se deroga la Directiva 2007/64/CE.

Bank Act, SC 1991, c 46, s 448.1(1).

\section{JURISPRUDENCIA CITADA}

Orionx SpA con Banco del Estado de Chile (2018): Corte Suprema, 3 de diciembre de 2018 (recurso de protección), disponible en: http://www.pjud.cl/documents/396729/0/ CRIPTOMONEDAS+SUPREMA.pdf/28b2de48-abb6-4173-8eac-c911177e018a. [Fecha de consulta: 2 de abril de 2019]. 
Toso Milos, Ángela "De-risking: una consecuencia indeseada del enfoque basado en el riesgo ..."

Surbtc SpA con Banco Itaú Corpbanca (2018): Corte de Apelaciones de Santiago, 25 de julio de 2018 (recurso de protección), disponible en: http://corte.poderjudicial.cl/ SITCORTEPORWEB/ [Fecha de consulta: 2 de abril de 2019].

ORIONX SPA CON BANCO DEL ESTADO DE ChILE (2018): Corte de Apelaciones de Santiago, 11 de julio de 2018 (recurso de protección), disponible en: http://corte.poderjudicial.cl/SITCORTEPORWEB/DownloadFile.do?TIP_Documento=3\&TIP_ Archivo=3\&COD_Opcion=1 \&COD_Corte=90\&CRR_IdTramite=22867108\&CRR_ IdDocumento=20288912 [Fecha de consulta: 2 de abril de 2019].

Cryptomkt Spa con Banco Scotiabank Chile S.A. (2018): Corte de Apelaciones de Santiago, 27 de junio de 2018 (recurso de protección), disponible en: http://corte.poderjudicial.cl/SITCORTEPORWEB/ [Fecha de consulta: 2 de abril de 2019].

Intervalores Corredores de Bolsa Limitada con Banco Bilbao Viscaya Argentaria Chile (2017): Corte de Apelaciones de Santiago, 4 de enero de 2017 (recurso de protección) en VLex, Código de búsqueda: VLEX-656836133, Fecha de consulta: 2 de abril de 2019.

BREDENKAMP V. STANDARDBANK, (4) SA 468 (SCA) (2010).

E.A.G.Y. CON BANCO SANTANDER-ChILE (2010): Corte de Apelaciones de Santiago, 10 de septiembre de 2010 (recurso de protección) en VLex, Código de búsqueda VLEX233669159, Fecha de consulta: 2 de abril de 2019.

E-TRANSINTERNATIONALFINANCELTD V. KIWIBANK LTD, 3 NZLR 241(2016).

HLONGWANEAND OtheRs V. ABSA BANK LiMITED AND ANOTHER, 75782/13, ZAGPPHC938 (2016).

V.L.C.M. CON BANCO ITAÚ (2016): Corte de Apelaciones de Santiago, 17 de octubre de 2016 (recurso de protección) en VLex, Código de búsqueda: VLEX-653061893, Fecha de consulta: 2 de abril de 2019.

J.A.L.F CON BANCO EDWARDS CITY (2014): Corte de Apelaciones de Valparaíso, 28 de abril de 2014 (recurso de protección) en VLex, Código de búsqueda: VLEX-580977286, Fecha de consulta: 2 de abril de 2019.

J.V.G.R. con BANCO DE CRÉdTo e InVERSIONES (2014): Corte Suprema de Santiago, 28 de agosto de 2014 (apelación recurso de protección) en VLex, Código de búsqueda: VLEX525960718, Fecha de consulta: 2 de abril de 2019.

Afex Transferencias y Cambios Ltda. y otro contra Banco de Chile (2013): Tribunal de Defensa de la Libre Competencia, sentencia No 129/2013, causa Rol C-201-10, 2 de mayo de 2013.

Dahabshitl TRANSFerSerVices LTD. V. BARCLAYSBANK PLC, EWHC 3379 (Ch) (2013). 